\title{
SPLITTING THE SCENE GRAPH \\ Using Spatial Relationship Graphs Instead of Scene Graphs in Augmented Reality
}

\author{
Florian Echtler, Manuel Huber, Daniel Pustka, Peter Keitler, Gudrun Klinker, PhD \\ Institut für Informatik I16, Technische Universität München, Boltzmannstr. 3, 85747 Garching \\ \{echtler,huberma,pustka,keitler,klinker\}@in.tum.de
}

\begin{abstract}
Keywords: scenegraph, spatial relationship graph, augmented reality, tracking
Abstract: $\quad$ Scene graphs have been a core element of 3D graphics since the publication of Inventor. However, in Virtual and Augmented Reality applications, 3D graphics are often interleaved with and controlled by real-world data provided by pose trackers, cameras and other kinds of sensors. In such a setup, the generalized concept of a Spatial Relationship Graph (SRG) might be better suited as an underlying data structure to describe the application and its components. In this paper, we will give an overview of the SRG concept, describe its difference to a scene graph and provide an example AR application built upon an SRG-based tracking library.
\end{abstract}

\section{INTRODUCTION AND RELATED WORK}

At least since the publication of Inventor (Wernecke, 1994), just about every major graphics system has been using scene graphs as the underlying data structure for describing a 3D world. Some widely-known examples include OpenSG (Reiners, 2002), OpenSceneGraph (Burns and Osfield, 2004) or Java3D (Sowizral et al., 1997). One common feature of all these systems is that they do not use a generalized graph, but rather a tree or a directed acyclic graph (DAG).

While this concept has proven highly useful for rendering large 3D worlds, augmented reality applications (Azuma, 1995) could benefit from a different approach. Although scenegraph-based APIs such as Studierstube (Schmalstieg et al., 2000) or OSGART (Looser et al., 2006) have been extended in ingenious ways to enable a wide selection of visualization concepts, we suggest that a variant of the scene graph, the spatial relationship graph (SRG), is a better-suited data structure in augmented reality applications.

A classical scene graph contains no representation for any real-world objects. Consequently, if one or more transformations in the scene are determined by external data sources, these transformations change "out of the blue", i.e. are influenced by objects not contained in the scene graph. This leads to a split in the information necessary to describe the application. The 3D graphics and their relations are described in the scene graph, while trackers, cameras etc. are described in another, possibly hardcoded, part of the application.

The SRG, on the other hand, is a graph which does not only describe spatial relations between virtual objects, but also between real-world objects such as cameras or pose trackers that are necessary for AR. SRGs have been introduced by (Newman et al., 2004; Wagner, 2005) and further explored by (Pustka et al., 2006).

A growing trend in many application fields is the move away from monolithic, compiled code towards high-level description languages. Such high-level languages provide, e.g., better maintainability and faster prototyping. SRGs and the UTQL language (Pustka et al., 2007) are able to provide such a high-level description for augmented reality applications by encapsulating the scene graph in a wrapper which allows a unified view of the entire setup. 


\section{SPATIAL RELATIONSHIP GRAPHS}

An SRG is a directed graph in which nodes represent coordinate systems. An edge represents a transformation between the nodes which it connects. This can, e.g., be a $6 \mathrm{D}$ pose for transforming between a tracker's world coordinate system and the tracked object. It could also be a projective transformation from $3 \mathrm{D}$ to $2 \mathrm{D}$ coordinates or a $2 \mathrm{D}$ feature position in an image.

An edge is labeled with attributes. These can be, e.g., the type of transformation the edge represents and whether it is a static or dynamic transformation, i.e. is fixed or changes over time. Usually, the static transformations are known in advance, for example a camera calibration.

Ultimately, the goal in most AR applications is to compute one or more previously unknown transformations and use these to render an augmentation. The SRG provides an intuitive way to gather the required information. Suppose we want to calculate the unknown transformation from coordinate system A to system D (see figure 1). The SRG does not contain a corresponding edge as this transformation is not yet known. However, if there is a path from A to D, say via the nodes $B$ and $C$, that consists of known transformations, then the concatenation of the three transformations $A \rightarrow B, B \rightarrow C$ and $C \rightarrow D$ results in the previously unknown transformation $A \rightarrow D$. Note that

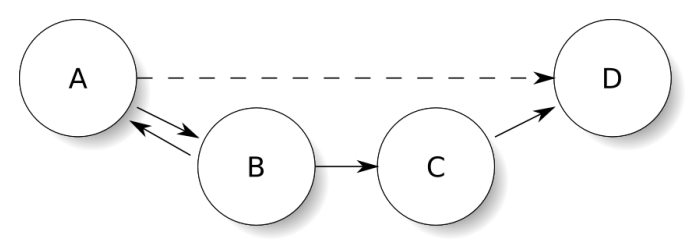

Figure 1: Two possible paths from A to D

if a transformation $A \rightarrow B$ is invertible (a 6D pose, for example), the inverse edge $B \rightarrow A$ can also be added to the graph. After inserting this new edge into the SRG, it can be used in subsequent calculations until the required transformation has been computed. In (Pustka et al., 2006), several more complex variants of such graph relationships, also called spatial relationship patterns, are described.

\section{AUGMENTED REALITY WITH SRGS}

In this section, we present a typical augmented reality application and its representation as an SRG as an example of how this concept can be used not only for pure tracking problems, but also for the rendering aspect of AR applications. Our setup consists of a camera and several optical markers, each of which is to be augmented with a different 3D graphics object.

As a consequence, the geometric relationship between the poses of the virtual objects depends on a number of independently controllable (tangible) real objects. The corresponding transformations in the virtual part of the SRG are not fixed.

The video image from the camera is to be displayed in the background. The resulting SRG is presented in figure 2 and shall be described in this section.

The conventions used in this example are as follows: Static edges are solid, while dynamic edges are dashed. Each edge is labeled with the type of transformation it represents. Edges which are labeled in brackets are not yet known and have to be computed from other edges.

\subsection{System and SRG Description}

The core of this setup is formed by a real-world camera. This camera introduces two coordinate systems: first, the 3D camera coordinate system, which usually has the $\mathrm{z}$-axis pointing along the view direction of the camera, and second, the 2D image plane coordinate system. We assume that the transformation between these two, a projection matrix labeled $P_{C}$ in the graph, is known through a prior camera calibration.

In addition to the camera, an optical flat marker is present in our setup. Assuming a marker of known size, four 2D point correspondences are necessary to calculate the pose of the marker relative to the camera. These correspondences are themselves transformations between the camera image coordinate system and the marker coordinate system and are labeled $2 D^{4}$ in the graph. The superscript 4 is used to denote a multi-edge, consisting of four distinct transformations.

In order to create a spatially aligned augmentation, we need to take several virtual coordinate systems into account. In this simple case, there exists a correspondence between each of the real-world coordinate systems and a virtual one. These correspondences are labeled with $i d$, denoting the identity transformation. 


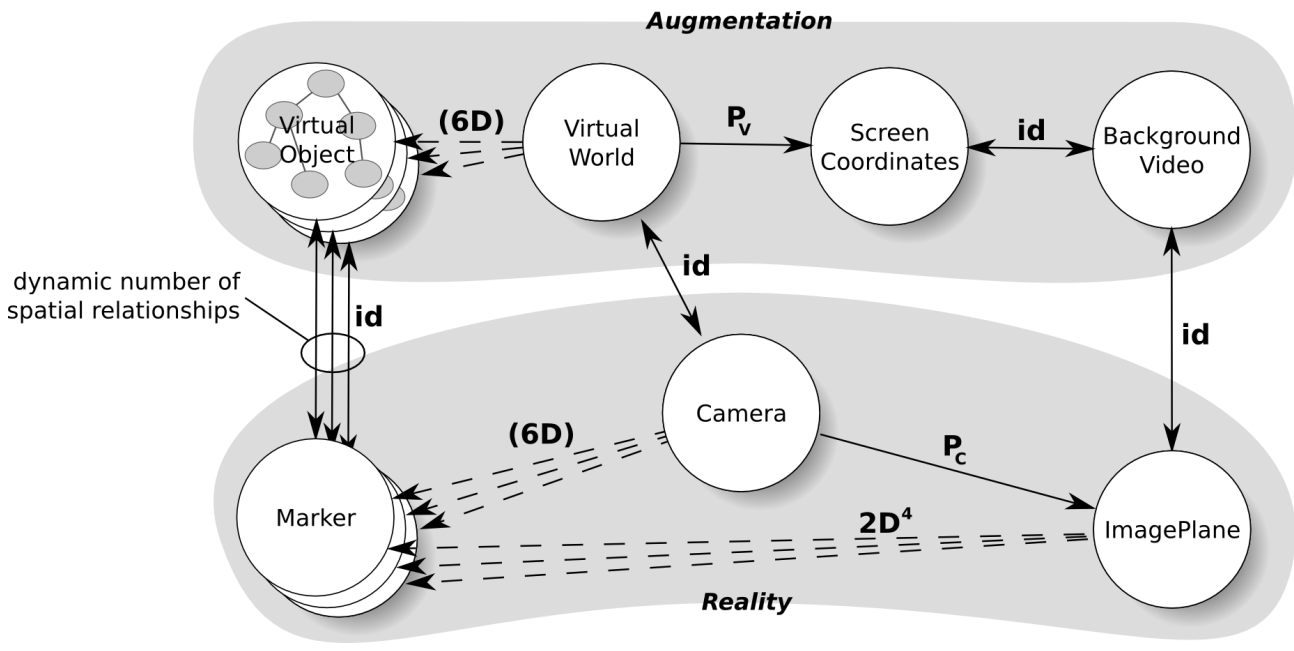

Figure 2: Example SRG for Rendering in AR

Special attention should be paid to the VirtualObject node. Within each such node, a full scenegraph-based description of graphical detail can be encapsulated. In figure 2, this is exemplified by a secondary tree structure inside the node. This encapsulating node is equivalent to the root of the scene graph. While all other scene graph nodes describing the object could also be integrated into the SRG, this would likely cause performance problems for graph algorithms operating on the SRG. Of course, a more complex AR application is bound to contain more than one virtual object. In this case, several encapsulated scene graphs exist and are traversed in turn when the corresponding object is rendered. If several identical objects are required, the same scene graph can even be reused and traversed from different contexts as described, e.g., by (Reitmayr, 2005).

In order to render the object at the correct position, the transformation labeled $(6 D)$ between the VirtualWorld and VirtualObject nodes must be known. By following the two identity transformations to the Marker and Camera nodes, we can infer that these two coordinate systems are linked by the same transformation. As we assume the camera calibration $P_{C}$ to be known, we can follow the path via the ImagePlane node and finally calculate the required $6 \mathrm{D}$ pose from the aforementioned four 2D-2D correspondences. Note that the Marker coordinate system is in fact a three-dimensional coordinate system, as we want to calculate a full $6 \mathrm{D}$ transformation to the Camera system. Therefore, we are dealing with 2D-3D correspondences. However, as the marker is known to be flat, the third coordinate can be defined as zero in all cases, reducing the correspondences to 2D-2D. ${ }^{1}$

In order to create a credible augmentation, the video image has to be integrated into our system if no optical see-through display is available. To achieve this, we introduce a node BackgroundVideo which is coplanar with the camera image plane. This node is also coplanar with the final screen coordinate system of the resulting image which is to be displayed to the user. Note that a loop of fixed transformations exists along the five nodes VirtualWorld, Camera, ImagePlane, BackgroundVideo and ScreenCoordinates. In order to be consistent with the previously described constraints, each path between two of these nodes must result in the same overall transformation. It therefore follows that the projection matrix used by the rendering system $P_{V}$ has to be equal to the camera projection matrix $P_{C}$. Otherwise, the field of view for the augmentation would differ from the video image, leading to disparities between the augmented and the real-world view.

\subsection{Notes on Implementation}

Our example is built on top of the Ubitrack (Huber et al., 2007) library. As this library's main focus re-

\footnotetext{
${ }^{1}$ This dimensional reduction could also be represented in the SRG by introducing an additional 2D coordinate system for each marker and a transformation which embeds this plane into the corresponding 3D Marker system. However, it was left out in the example SRG to avoid unnecessary complexity.
} 


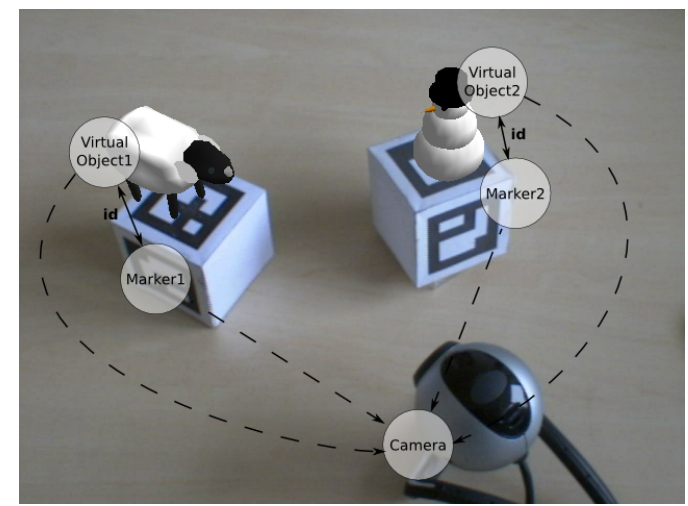

Figure 3: augmented optical markers and corresponding SRG nodes

mains on sensor fusion, we decided against integration with one of the large scene graph APIs mentioned above in order to keep the library's dependency count low. Instead, we implemented a basic scene graph traversal based on the XML visitor pattern already present in the library, taking advantage of the inherent scene graph structure of X3D files. This allows implementation of a small scene graph API in about $10 \mathrm{kB}$ of $\mathrm{C}++$ code to provide a basic visualization of encapsulated 3D objects with almost no overhead.

Only if any of the edges incident to the VirtualWorld node changes, the view has to be rerendered. The implementation updates the transformations to each VirtualObject and traverses each encapsulated scene graph in turn, thereby avoiding unnecessary redraws. In our example, which does not contain interpolators, this results in a synchronization of the rendered output to the camera frame rate. In figure 3, our example scenario is shown from a second camera's perspective, along with the directly visible SRG nodes.

\section{CONCLUSION AND OUTLOOK}

We propose that for augmented reality applications, the spatial relationship graph is a better-suited data structure than a scene graph. While the scene graph is highly useful for pure rendering tasks, the SRG presents a more intuitive way to incorporate the various real-world coordinate systems which invariably are part of AR scenarios. We therefore suggest to split the scene graph into several static subtrees and encapsulate each of those in an SRG node, thereby creating an unified view of the entire AR application.

\section{ACKNOWLEDGEMENTS}

This work was supported in part by a grant from Bayerische Forschungsstiftung (BFS) for the TrackFrame project and in part by the PRESENCCIA project.

\section{REFERENCES}

Azuma, R. (1995). A survey of augmented reality. In $S I G$ GRAPH' 95 Proceedings (Aug. 1995), pp. 1-38.

Burns, D. and Osfield, R. (2004). Open scene graph - a: Introduction, b: Examples and applications. In $V R$ '04: Proceedings of the IEEE Virtual Reality 2004 (VR'04), page 265.

Huber, M., Pustka, D., Keitler, P., Echtler, F., and Klinker, G. (2007). A System Architecture for Ubiquitous Tracking Environments. In Proceedings of the 6th International Symposium on Mixed and Augmented Reality (ISMAR).

Looser, J., Grasset, R., Seichter, H., and Billinghurst, M. (2006). OSGART - A Pragmatic Approach to MR. In Industrial Workshop at ISMAR 2006.

Newman, J., Wagner, M., Bauer, M., MacWilliams, A.; Pintaric, T., Beyer, D., Pustka, D., Strasser, F., Schmalstieg, D., and Klinker, G. (2-5 Nov. 2004). Ubiquitous tracking for augmented reality. Mixed and Augmented Reality, 2004. ISMAR 2004. Third IEEE and ACM International Symposium on, pages 192-201.

Pustka, D., Huber, M., Bauer, M., and Klinker, G. (2006). Spatial Relationship Patterns: Elements of Reusable Tracking and Calibration Systems. In Proc. IEEE International Symposium on Mixed and Augmented Reality (ISMAR'06).

Pustka, D., Huber, M., Echtler, F., and Keitler, P. (2007). UTQL: The Ubiquitous Tracking Query Language v1.0. Technical Report TUM-I0718, Institut für Informatik, Technische Universität München.

Reiners, D. (2002). Open SG: A Scene Graph System for Flexible and Efficient Realtime Rendering for Virtual and Augmented Reality Applications. PhD thesis.

Reitmayr, G.; Schmalstieg, D. (2005). Flexible parametrization of scene graphs. Virtual Reality, 2005. Proceedings. VR 2005. IEEE, pages 51-58.

Schmalstieg, D., Fuhrmann, A., Hesina, G., Szalavári, Z., Encarnação, L. M., Gervautz, M., and Purgathofer, W. (2000). The Studierstube Augmented Reality Project. Technical report, Institute of Computer Graphics and Algorithms, Vienna University of Technology.

Sowizral, H., Rushforth, K., and Deering, M. (1997). The Java 3D API Specification.

Wagner, M. (2005). Tracking With Multiple Sensors. PhD thesis.

Wernecke, J. (1994). The Inventor Mentor: Programming Object-Oriented $3 D$ Graphics with Open Inventor. 